\title{
Transition-Metal Nanoparticle Oxidation in a Chemically Nonhomogenous Environment Revealed by 2p3d Resonant X-ray Emission
}

\author{
Matti M. van Schooneveld, ${ }^{*}{ }^{\dagger}$ Edlira Suljoti, ${ }^{\dagger, \S}$ Carlos Campos-Cuerva, ${ }^{\dagger}$ Robert W. Gosselink, ${ }^{\dagger}$ \\ Ad M. J. van der Eerden, ${ }^{\dagger}$ Justine Schlappa, ${ }^{\ddagger}, \S$ Kejin J. Zhou, ${ }^{\ddagger}$ Claude Monney, ${ }^{\ddagger}$ Thorsten Schmitt, ${ }^{\ddagger}$ \\ and Frank M. F. de Groot*, \\ ${ }^{\dagger}$ Inorganic Chemistry \& Catalysis, Debye Institute for Nanomaterials Science, Utrecht University, Universiteitsweg 99, 3584 CG, The \\ Netherlands \\ ${ }^{\ddagger}$ Paul Scherrer Institut (PSI), Swiss Light Source, CH-5232 Villigen, Switzerland
}

\section{Supporting Information}

ABSTRACT: X-ray absorption spectroscopy (XAS) is often employed in fields such as catalysis to determine whether transition-metal nanoparticles are oxidized. Here we show $2 \mathrm{p}_{3 / 2}$ XAS and 2p3d resonant X-ray emission spectroscopy (RXES) data of oleate-coated cobalt nanoparticles with average diameters of 4.0, 4.2, 5.0, 8.4, and $15.2 \mathrm{~nm}$. Two particle batches were exposed to air for different periods of time, whereas the others were measured as synthesized. In the colloidal nanoparticles, the cobalt sites can have different chemical environments (metallic/oxidized/ surface-coordinated), and it is shown that most XAS data cannot distinguish whether the nanoparticles are oxidized or surface-coated. In contrast, the high-energy resolution RXES spectra reveal whether more than the first metal layer is oxidized based on the unique energetic

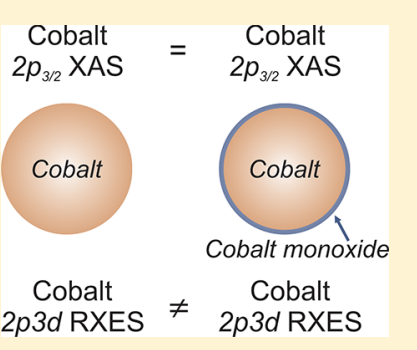
separation of spectral features related to the formal metal (X-ray fluorescence) or to a metal oxide (d-d excitations). This is the first demonstration of metal 2p3d RXES as a novel surface science tool.

SECTION: Spectroscopy, Photochemistry, and Excited States

\begin{abstract}
T $\mathrm{n}$ many fields of research where metallic nanoparticles are 1 applied, it is often relevant to know their degree of oxidation. In catalysis, the difference between a metal oxide and a metal nanoparticle system can mean the difference between an active or inactive catalyst. It is also known that the gas atmosphere or liquid environment of a catalytically active surface alters the surface chemical potential and that in situ studies are essential to understand the catalyst's mechanism. ${ }^{1}$ X-ray spectroscopies offer the important advantage of element selectivity; therefore, several in situ X-ray photoemission spectroscopy (XPS) and/or $\mathrm{X}$-ray absorption spectroscopy (XAS) studies have been performed to investigate metal nanoparticle oxidation under conditions relevant to the reaction. ${ }^{2-8}$ In principle, both the $2 \mathrm{p}$ XPS and the 2p XAS spectra of transition metals are highly useful in metal characterization due to the large many-electron multiplet effects at this edge, where we note that the screening effects induced in XPS due to the removal of a photoelectron make the technique less sensitive to subtle chemical differences such as the ligand or crystal environment around a metal ion. $^{9-11}$ In 2 p XAS, such multiplet splitting is more substantial, which allows for a significant degree of understanding through atomic, ligand field, and charge transfer multiplet theories. ${ }^{12}$ Although 2p XAS is a widely available tool nowadays, an intrinsic problem of applying the technique to chemically heterogeneous systems, such as real catalysts, is that (a) the spectra of atomic or ionic species in different chemical environments overlap in energy and (b) the same spectrum
\end{abstract}

can theoretically often be interpreted by assuming different chemical environments. ${ }^{13}$ Here we use colloidal cobalt nanoparticles as model systems that capture part of the complexity of a heterogeneous catalyst. After all, the cobalt inside and at the surface of the nanoparticles, where cobalt sites are coated with surfactant molecules, possesses different chemical environments, whereas in a real heterogeneous catalyst the nanoparticle support material, promoters, and cocatalysts add multiple dimensions to chemical heterogeneity. ${ }^{14}$ We show here that for nanoparticles in chemically nonhomogenous environments, high-resolution $2 \mathrm{p} 3 \mathrm{~d}$ resonant $\mathrm{X}$-ray emission spectroscopy (RXES; also known as resonant inelastic X-ray scattering (RIXS)) is more sensitive to metal oxidation than 2p XAS and that care should be taken with conclusions drawn on the basis of XAS alone. We note that recently fluorescence-detected 1s XAS spectra acquired from RXES 1s2p $(\mathrm{K} \beta)$ emission lines were also used to study oxidized cobalt particles in detail, ${ }^{15}$ but this is the first study to report metal $2 \mathrm{p} 3 \mathrm{~d}$ RXES as a surface science tool. The fact that (a) 2p3d RXES is capable of characterizing the electronic structure of relatively complex transition metal systems and that (b) the number of high-resolution soft RXES instruments will

Received: February 5, 2013

Accepted: March 22, 2013

Published: March 22, 2013 
Table 1. Average Nanoparticle Diameter and Elemental Composition of the Studied Systems

\begin{tabular}{|c|c|c|}
\hline compound & TEM diameter $(\mathrm{nm}) \pm$ polydispersity $^{a}$ & composition \\
\hline I & $4.2 \pm 11.9 \%$ & oxidized $\varepsilon$-Co/OA/TOPO \\
\hline II & $4.0 \pm 8.8 \%$ & oxidized $\varepsilon$-Co/OA/TOPO \\
\hline III & $8.4 \pm 14.1 \%$ & $\varepsilon$-Co/OA/TOPO \\
\hline IV & $5.0 \pm 13.7 \%$ & $\varepsilon$-Co/OA/TOPO \\
\hline $\mathbf{V}$ & $15.2 \pm 17.5 \%$ & $\mathrm{fcc} \mathrm{Co} / \mathrm{TOPO}$ \\
\hline
\end{tabular}
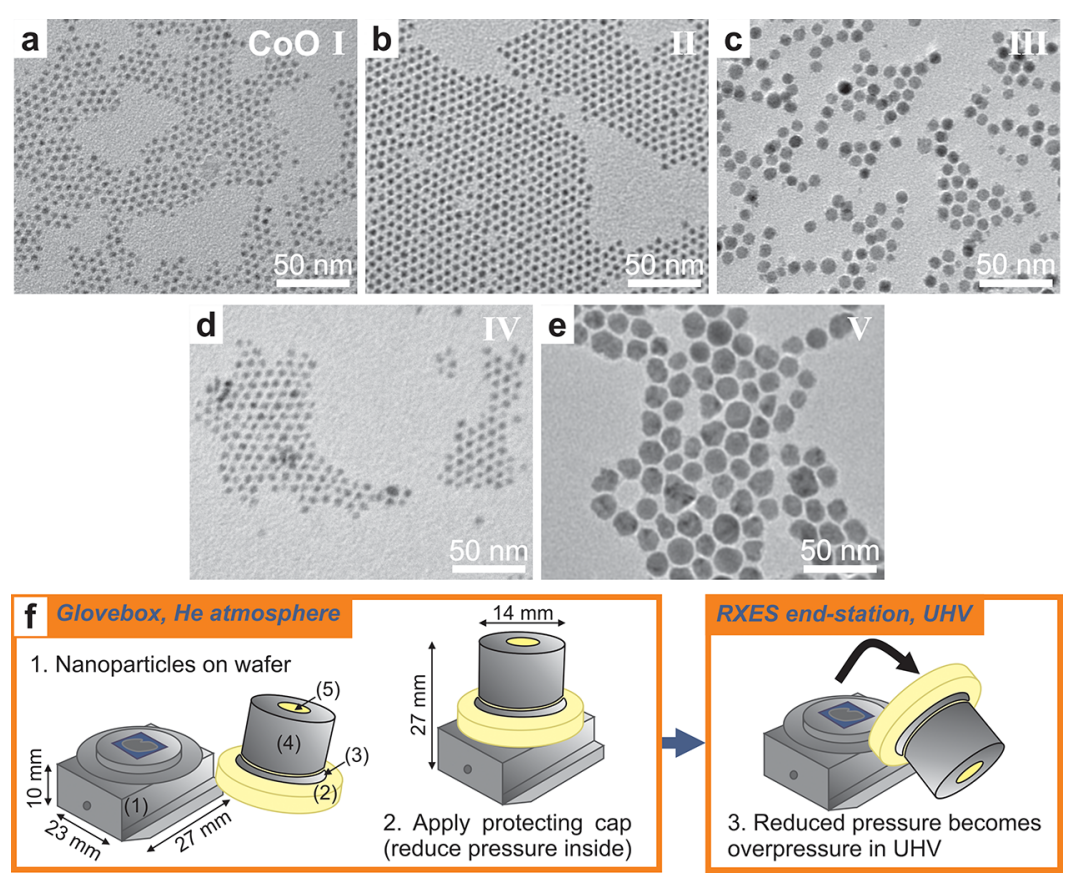

Figure 1. (a-e) TEM micrographs of nanoparticle systems $I-V$ and (f) an artistic impression of the tool that allows sample transfer in an inert environment to the RXES endstation at the ADRESS beamline.

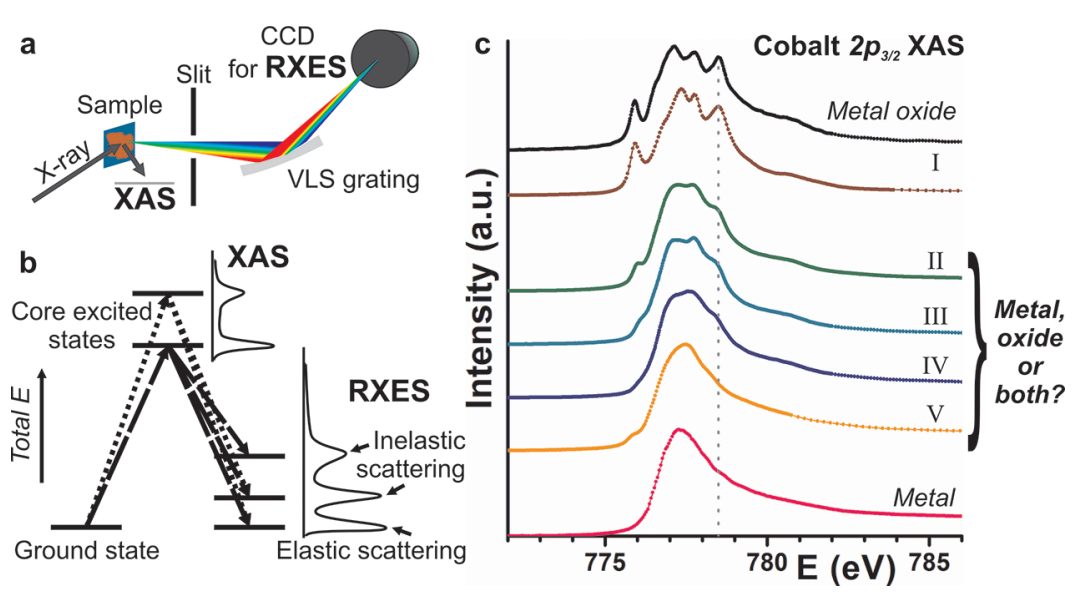

Figure 2. Schematic representations of the $2 p$ XAS and 2p3d RXES (a) experimental setup and (b) processes. (c) Experimental cobalt $2 p_{3 / 2}$ XAS spectra of nanoparticle batches I-V and literature reference spectra of cobalt metal ${ }^{25}$ and cobalt monoxide powder. ${ }^{22}$ The dotted gray line at 778.4 $\mathrm{eV}$ indicates the excitation energy of the 2p3d RXES spectra of Figure 3.

increase rapidly in the next years ${ }^{16}$ promises the advent of important insights from in situ synchrotron-based catalytic studies during actual reactions.

Five different cobalt nanoparticle systems, I-V, were synthesized through published, hot-injection synthesis methods and characterized by transmission electron microscopy (TEM) and $\mathrm{X}$-ray powder diffraction (XRD), as described in detail in the Supporting Information. Their sizes and compositions are given in Table 1.

Systems I-IV consist of $\varepsilon$-Co ${ }^{17}$ nanoparticles prepared by the Puntes method. ${ }^{18}$ System V consists of fcc Co nanoparticles made through a slightly adapted method that used acetonated cobalt carbonyl. ${ }^{19}$ For systems I-IV, the nanoparticles were synthesized in the presence of mixtures of trioctylphosphine 


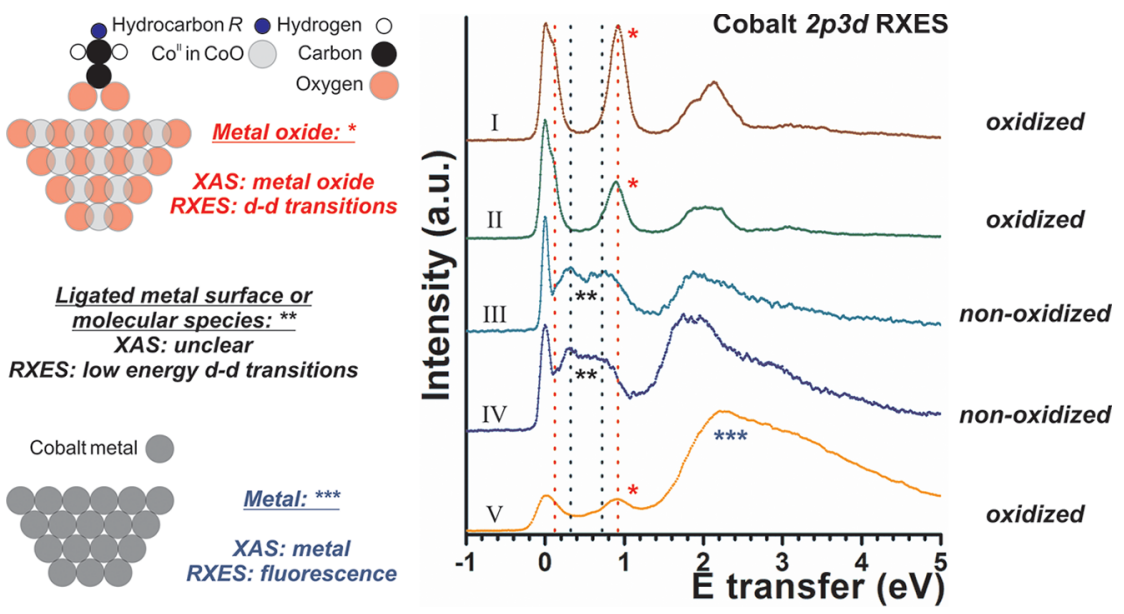

Figure 3. Experimental cobalt 2p3d RXES spectra of I-V (right). To the left, a schematic representation of a ligated metal oxide substrate is shown, together with a pure metal substrate. One red asterisk and red dotted lines in the spectra indicate $\mathrm{d}$-d excitations from bulk cobalt monoxide. Two black asterisks and black dotted lines indicate low-energy $\mathrm{d}$-d excitations present in systems of nonoxidized cobalt nanoparticles. Three blue asterisks indicate X-ray fluorescence due to pure cobalt metal.

oxide (TOPO) and oleic acid (OA). When both molecules are present in the used ratios, the TOPO acts as a kinetic growth controller, ${ }^{20}$ whereas the $\mathrm{OA}$ is the main capping agent. ${ }^{21}$ For system V, only TOPO was used to obtain larger cobalt nanoparticles. These had an fcc crystal structure. The particles were all synthesized in an inert atmosphere. When the $\varepsilon$-Co nanoparticles are exposed to air they oxidize. ${ }^{22}$ When large nanoparticles are used, a passivation $\mathrm{CoO}$ layer forms around the particles. This is revealed in Figure S1 in the Supporting Information by time-resolved XRD studies. Systems I and II were both exposed to air on purpose for 3 weeks and a few hours, respectively. Systems III-V were not deliberately airexposed. TEM images of the nanoparticles are shown in Figure $1 \mathrm{a}-\mathrm{e}$.

For the $2 \mathrm{p}$ XAS and 2p3d RXES measurements, the nanoparticles were transferred to the measurement chamber of the beamline in an inert environment to measure the nanoparticles as synthesized and washed (systems III-V) or as synthesized, washed, and oxidized (systems I-II). Figure If depicts a tool that we designed for this purpose. The tool is optimized for the RXES endstation at the ADRESS beamline of the SLS. ${ }^{23,24}$ In short, a protective cap is sucked over the sample by creating some underpressure inside the cap's cavity. This is done inside an inert-atmosphere glovebox. After transfer to the load lock chamber of the RXES setup, the chamber is evacuated and the underpressure becomes an overpressure, thereby releasing the cap and unlocking the sample for measurements. (For employment detail, see the Supporting Information).

Figure 2a sketches the experimental setup of the ADRESS beamline at the SLS synchrotron. Here cobalt $2 p$ XAS spectra and cobalt 2p3d RXES spectra of the nanoparticles were obtained (acquisition details specified in the Supporting Information). ${ }^{2,26-28}$ The XAS spectra are measured in total electron yield (TEY) mode. RXES spectra are measured using a variable line spacing (VLS) grating and a liquid-nitrogen-cooled charge-coupled device (CCD) camera.

Figure 2b schematically illustrates the XAS and RXES transition processes. In metal $2 p$ XAS, X-ray photons induce electron transitions from occupied $2 p$ states to the empty density of 3d-states of the metal system. In 2p3d RXES, one collects photons that are emitted when electrons from $3 \mathrm{~d}$ states subsequently decay radiatively to the now empty $2 p$ core levels. A decaying electron may be the same as the electron that was excited in the XAS process. In this case, no energy is transferred and the emitted energy is equal to the incident photon energy. This is an elastic RXES event. If, however, the energy of the emitted X-ray photon is lower than that of the incident one, an inelastic RXES event occurred. In a 2p3d RXES experiment, inelastic events with energies up to $20 \mathrm{eV}$ can be detected. Two types of inelastic events exist: resonant Raman scattering and Xray fluorescence. In resonant Raman scattering, the transferred energy excites processes in the sample such as vibrations, phonons, magnons, d-d, and charge-transfer excitations. ${ }^{29-32}$ RXES signals of this type occur at a fixed transfer or loss energy irrespective of the incident X-ray energy. X-ray fluorescence occurs from excitations to continuum states. It has a fixed emission energy and hence a varying transferred energy in the inelastic RXES event irrespective of the incident X-ray energy.

Figure $2 \mathrm{c}$ shows the $2 \mathrm{p}_{3 / 2}$ XAS spectra of nanoparticle batches $\mathrm{I}-\mathrm{V}$. In addition, literature reference $2 \mathrm{p}_{3 / 2}$ XAS spectra of cobalt metal taken from Chen et al. $^{25}$ and of cobalt monoxide powder ${ }^{22}$ are shown. From the analysis, it is clear that the nanoparticles in system I are cobalt monoxide. For IIIV, the XAS spectra show weak fine structure and almost no fine structure for system V. From such analysis, V seems to consist of metallic cobalt, whereas for systems II-IV it is unclear whether the cobalt nanoparticles are oxidized because both cobalt oleate bonds, ${ }^{13}$ combinations of cobalt metal and cobalt oxide, or combinations of all species could yield such spectral fine structure. Whereas others have interpreted XAS spectra obtained on complex chemical systems with fitting procedures, we note that there is often no theoretical basis provided for the number and the type of model spectra included.

In Figure 3, we show the corresponding 2p3d RXES spectra. The excitation energy of $778.4 \mathrm{eV}$ at which the spectra were acquired is indicated in Figure 2c. The RXES spectrum of the deliberately oxidized cobalt nanoparticles of system I is indicative of cobalt monoxide. The origin of cobalt monoxide 2p3d RXES peaks was discussed in the context of ligand field multiplet (LFM) calculations. ${ }^{22}$ In short, cobalt monoxide has, among others, characteristic d-d excitations at 0.1 and $1 \mathrm{eV}$. Interestingly, system II also displays features at 0.1 and $1 \mathrm{eV}$, 
but they are less intense with respect to the elastic peak than for system I. Whereas system I was exposed to air for 3 weeks, system II was exposed for a few hours. This result suggests that 2p3d RXES is sensitive to the degree of nanoparticle oxidation, but this statement cannot be proven from the current data. Although both measurements were acquired using the same geometry and polarization, more quantitative measurements that consider other intensity-influencing parameters, such as the sample surface roughness, should be performed for this purpose.

Systems III and IV display two low-energy resonant Raman peaks at 0.3 and $0.75 \mathrm{eV}$. On the basis of LFM and timedependent density functional (TD-DFT) calculations, these peaks were interpreted to originate from ligated cobalt ions in the outer atomic layer of the nanoparticles or, alternatively, from coexisting cobalt molecular complexes. ${ }^{33}$ The peaks are in any case $d$-d excitations resulting from cobalt ions in a low symmetric ligand field with a coordination number maximum of four oxygen ions. ${ }^{33}$ Importantly, the presence of these peaks and the absence of the features at 0.1 and $1 \mathrm{eV}$ implies that the probed nanoparticles are nonoxidized or only their outer atomic layer is oxidized because they lack the d-d excitations for $\mathrm{Co}^{2+}$ in cobalt monoxide. It is important to realize that in case more than the first atomic cobalt nanoparticle layer would be oxidized, the number of cobalt atoms with a six-fold oxygen octahedral coordination (as is the case in cobalt monoxide) would rapidly dominate the presence of cobalt ions in a lower coordination environment. A surface with more than one cobalt layer oxidized will then give rise to the $\mathrm{d}$-d excitations at 0.1 and $1 \mathrm{eV}$, as shown for the cobalt monoxide particles in systems I and II. The fine structure in the $2 \mathrm{p}_{3 / 2}$ XAS spectra of these nanoparticle systems is thus also not a result of nanoparticle oxidation but of nanoparticle ligation or the existence of a minority of cobalt molecular complexes, the same possible effects that give rise to the peaks at 0.3 and $0.75 \mathrm{eV} .^{33}$

The sensitivity of $2 \mathrm{p} 3 \mathrm{~d}$ RXES to surface oxidation holds because a true metal exhibits no resonant Raman features but only strong X-ray fluorescence. System V is indicative of the typical 2p3d RXES spectra of a formal metal. These nanoparticles were made large on purpose $(15.2 \mathrm{~nm}$ diameter; see Table 1) so as to enhance the chance of obtaining a large fraction of formal cobalt. A strong X-ray fluorescence peak is observed around $2.2 \mathrm{eV}$ in its $2 \mathrm{p} 3 \mathrm{~d}$ RXES spectrum, which we relate to metallic cobalt. The absence of resonant Raman features in 2p3d RXES spectra of metallic cobalt was previously observed but not reported. ${ }^{34}$ Another example of typical $2 \mathrm{p} 3 \mathrm{~d}$ RXES behavior can be seen in Figure S2 in the Supporting Information for $2 \mathrm{p} 3 \mathrm{~d}$ RXES at the nickel $2 \mathrm{p}_{3 / 2}$ resonance in cobalt-nickel nanoparticles, where the nickel is fully metallic and the RXES spectra only show X-ray fluorescence.

The spectrum of system $\mathrm{V}$, however, also shows resonant Raman features at 0.1 and $1 \mathrm{eV}$, characteristic of cobalt monoxide. This reveals that although the $2 \mathrm{p}_{3 / 2}$ XAS spectrum of these materials indicates the particles to be metallic, the surface of the particles must be oxidized. Such interpretation of 2p3d RXES spectra is especially possible for RXES spectra acquired at excitation energies on the high-energy side of a $2 \mathrm{p}_{3 / 2}$ XAS spectrum because here the X-ray fluorescence occurs at higher transfer energies in the RXES spectra than the d-d features at low transfer energies; therefore, they are not hidden under the fluorescent signal.

The combination of the absence of $d$-d excitations in $2 \mathrm{p} 3 \mathrm{~d}$ RXES spectra of a formal metal and the clear relationship between ion coordination and energies of $\mathrm{d}$-d excitations ${ }^{13}$ results in an extraordinary sensitivity of $2 \mathrm{p} 3 \mathrm{~d}$ RXES with respect to surface oxidation of transition-metal substrates in general and transition-metal nanoparticles in particular. The surface sensitivity of RXES is thus based on fundamentally different principles than the surface sensitivity of (resonant) XPS. Metal 2p3d RXES is best compared with valence XPS, also called photoemission spectroscopy (PES), because both measure valence electronic states. (RXES, measures however, unoccupied and PES occupied states.) In XPS, it is the inelastic mean free path of the emitted electron that restricts XPS to probe the first $\sim 5 \mathrm{~nm}$ of the material when soft X-rays are used. $^{35}$ In contrast, RXES is a photon-in photon-out experiment in which the emitted photons can originate from a few micrometers underneath the surface of a material. However, metal 2p3d RXES is turned into a technique sensitive to surface oxidation because (1) the metal bulk of the nanoparticles shows only fluorescent decay that can be separated out of the low energy $d$-d regime and (2) oxidized metal ions in low oxygen coordination (first atomic surface layer incompletely oxidized) generate different $\mathrm{d}$-d states from fully oxidized metal ions. Whereas the binding energy of electrons from $\mathrm{Co}^{2+}$ versus $\mathrm{Co}^{0}$ changes 1.3 to $2.3 \mathrm{eV}$ in XPS as well, ${ }^{8}$ here the separation described under point (1) can be much larger and tuned as a function of the excitation energy. When considering $\mathrm{Co}^{2+}$ ions in different ligand fields, metal 2p3d RXES will have a superior discriminative power based on the appearance of characteristic ligand field split d-d excitations, ${ }^{13}$ whereas (valence band) XPS measures the electron emission spectrum that does not include these $\mathrm{d}$-d excitations.

For the here studied cobalt nanoparticles, RXES has a couple of additional advantages over XPS. The RXES signal comes from multiple layers of nanoparticles and from all sides of the nanoparticles (not only the sides facing the detector as in XPS). Furthermore, the relatively small electron inelastic mean free path in XPS generates problems when studying buried surfaces. Here the cobalt nanoparticles are coated with OA, which consists of an array of 18 carbon atoms that stick out from the nanoparticle surface. This implies that the electrons have to travel through $\sim 2 \mathrm{~nm}$ organic material, which drastically lowers the signal. A related advantage of RXES is thus that is allows measurements at 1 bar reactor conditions by the use of a membrane between the detector and the sample.

In a broader context, it was stated that it is essential to investigate chemical reactions during in situ conditions. ${ }^{1}$ Here we add to the discussion that it is equally important to perform data analysis that is based on sufficient theoretical insight into the information content of a spectrum. The main advantage of $2 p 3 d$ RXES over $2 p$ XAS and $2 p$ XES in this respect is that (1) the spectral interpretation is more unique due to a combination of tunable energy separation of certain features, less multiplet states, and a better spectral resolution ${ }^{13}$ and (2) it does not require fitting procedures. Fitting procedures based on nonunique model spectra can yield wrong conclusions. The technique therefore allows understanding of more complex systems, as shown in the present study. Together with the rising number of available high-resolution RXES beamlines ${ }^{16}$ and the combination with other RXES experiments, such as O 1s2p RXES, the technique will become an important analytical tool for catalytic, fuel cell, and battery studies.

In conclusion, $2 \mathrm{p}_{3 / 2}$ XAS and $2 \mathrm{p} 3 \mathrm{~d}$ RXES spectra on five different oleate-coated cobalt nanoparticle systems were presented. The $2 \mathrm{p}_{3 / 2}$ XAS spectra show weak fine structure 
that differs from the spectra of formal cobalt metal or cobalt monoxide. It is unclear from such spectra if the cobalt nanoparticles are oxidized or metallic because cobalt oleate bonds are also present. Corresponding high-energy resolution 2p3d RXES spectra allow for the discrimination of oxidized from nonoxidized cobalt nanoparticles. This is the first study where metal 2p3d RXES is used as a surface science tool, making use of the observation that for purely metallic materials, metal 2p3d RXES shows no d-d excitations. Cobalt nanoparticle oxidation gives rise to characteristic $\mathrm{d}$-d features at 0.1 and $1 \mathrm{eV}$ if more than the first atomic surface layer is oxidized to cobalt monoxide. If only low-coordinated cobalt ions are present, as a result of surface ligation or incomplete oxidation of the first cobalt monolayer, then low-energy d-d excitations exist. As an example of the oxidation sensitivity of $2 \mathrm{p} 3 \mathrm{~d}$ RXES, $15 \mathrm{~nm}$ large cobalt nanoparticles that are concluded to be metallic from the $2 \mathrm{p}_{3 / 2}$ XAS spectra alone, are shown to be surface oxidized when considering their 2 p3d RXES spectra.

\section{ASSOCIATED CONTENT}

\section{S Supporting Information}

Experimental section describing nanoparticle syntheses, characterization, and XAS/RXES acquisition. Description of transfer tool for XAS/RXES measurements of atmospheresensitive samples. Additional XAS/RXES data on CoNi alloy nanoparticles. This material is available free of charge via the Internet at http://pubs.acs.org.

\section{AUTHOR INFORMATION}

\section{Corresponding Author}

*E-mail: Matti.van-Schooneveld@cec.mpg.de (M.M.v.S.) and F.M.F.deGroot@uu.nl (F.M.F.d.G.).

\section{Present Address}

${ }^{\S}$ Edlira Suljoti and Justine Schlappa: Institute Methods and Instrumentation for Synchrotron Radiation Research, Helmholtz Zentrum Berlin, Hahn-Meitner-Platz 1, 14109 Berlin, Germany.

\section{Notes}

The authors declare no competing financial interest.

\section{ACKNOWLEDGMENTS}

We thank V. N. Strocov for beamline support and V. R. Calderone and J. Pet for discussions and help with nanoparticle synthesis, respectively. This work was financially supported by a VICI grant (FMFdG) of The Netherlands Organization for Scientific Research (NWO-CW).

\section{REFERENCES}

(1) Schlögl, R.; Knop-Gericke, A.; Hävecker, M.; Wild, U.; Frickel, D.; Ressler, T.; Jentoft, R. E.; Wienold, J.; Mestl, G.; Blume, A.; Timpe, O.; Uchida, Y. In Situ Analysis of Metal-Oxide Systems Used for Selective Oxidation Catalysis: How Essential Is Chemical Complexity? Top. Catal. 2001, 15, 219-228.

(2) Herranz, T.; Deng, X.; Cabot, A.; Guo, J. H.; Salmeron, M. Influence of the Cobalt Particle Size in the CO Hydrogenation Reaction Studied by In Situ X-Ray Absorption Spectroscopy. J. Phys. Chem. B 2009, 113, 10721-10727.

(3) Alayoglu, S.; Beaumont, S. K.; Zheng, F.; Pushkarev, V. V.; Zheng, H.; Iablokov, V.; Liu, Z.; Guo, J.; Kruse, N.; Somorjai, G. A. $\mathrm{CO}_{2}$ Hydrogenation Studies on Co and CoPt Bimetallic Nanoparticles Under Reaction Conditions Using TEM, XPS and NEXAFS. Top Catal. 2011, 54, 778-785.
(4) Wiedwald, U.; Fauth, K.; Heßler, M.; Boyen, H. G.; Weigl, F.; Hilgendorff, M.; Giersig, M.; Schütz, G.; Ziemann, P.; Farle, M. From Colloidal Co/CoO Core/Shell Nanoparticles to Arrays of Metallic Nanomagnets: Surface Modification and Magnetic Properties. ChemPhysChem 2005, 6, 2522-2526.

(5) Zheng, F.; Alayoglu, S.; Guo, J.; Pushkarev, V.; Li, Y.; Glans, P.A.; Chen, J.-L.; Somorjai, G. In-situ X-ray Absorption Study of Evolution of Oxidation States and Structure of Cobalt in Co and CoPt Bimetallic Nanoparticles $(4 \mathrm{~nm})$ under Reducing $\left(\mathrm{H}_{2}\right)$ and Oxidizing $\left(\mathrm{O}_{2}\right)$ Environments. Nano Lett. 2011, 11, 847-853.

(6) Zheng, F.; Alayoglu, S.; Pushkarev, V. V.; Beaumont, S. K.; Specht, C.; Aksoy, F.; Liu, Z.; Guo, J.; Somorjai, G. A. In Situ Study of Oxidation States and Structure of $4 \mathrm{~nm}$ CoPt Bimetallic Nanoparticles During CO Oxidation Using X-ray Spectroscopies in Comparison with Reaction Turnover Frequency. Catal. Today 2012, 182, 54-59.

(7) Papaefthimiou, V.; Dintzer, T.; Dupuis, V.; Tamion, A.; Tournus, F.; Teschner, D.; Hävecker, M.; Knop-Gericke, A.; Schlögl, R.; Zafeiratos, S. When a Metastable Oxide Stabilizes at the Nanoscale: Wurtzite CoO Formation upon Dealloying of PtCo Nanoparticles. J. Phys. Chem. Lett. 2011, 2, 900-904.

(8) Papaefthimiou, V.; Dintzer, T.; Dupuis, V.; Tamion, A.; Tournus, F.; Hillion, A.; Teschner, D.; Hävecker, M.; Knop-Gericke, A.; Schlögl, R; et al. Nontrivial Redox Behavior of Nanosized Cobalt: New Insights from Ambient Pressure X-ray Photoelectron and Absorption Spectroscopies. ACS Nano 2011, 5, 2182-2190.

(9) Cowan, R. D. The Theory of Atomic Structure and Spectra; University of California Press: Berkeley, CA, 1981.

(10) Jørgensen, C. K. Absorption Spectra and Chemical Bonding in Complexes; Pergamon: Oxford, U.K., 1962.

(11) Slater, J. C. The Theory of Complex Spectra. Phys. Rev. 1929, 34, 1293-1322.

(12) de Groot, F. M. F.; Kotani, A. Core Level Spectroscopy of Solids; CRC Press: Boca Raton, FL; 2008.

(13) van Schooneveld, M. M.; Gosselink, R. W.; Eggenhuisen, T. M.; Al Samarai, M.; Monney, C.; Zhou, K. J.; Schmitt, T.; de Groot, F. M. F. A Multispectroscopic Study of 3d Orbitals in Cobalt Carboxylates: The High Sensitivity of 2p3d Resonant X-ray Emission Spectroscopy to the Ligand Field. Angew. Chem., Int. Ed. 2012, 52, 1170-1174.

(14) de Jong, K. P. Synthesis of Solid Catalysts; Wiley-VCH: Weinheim, Germany; 2009.

(15) Kühn, T.-J.; Caliebe, W.; Matoussevitch, N.; Bönnemann, H.; Hormes, J. Site-Selective X-ray Absorption Spectroscopy of Cobalt Nanoparticles. Appl. Organomet. Chem. 2011, 25, 577-584.

(16) The number of beamlines with the required resolution is at this moment limited to five, respectively, SLS-ADRESS, Spring8BL07LSU, Soleil-sextants, NSRRC-I511, and CLS 10ID-2. In the next years, further beamlines will become available at Diamond, ESRF, BESSY, NSLS2, and ALS.

(17) Dinega, D. P.; Bawendi, M. G. A Solution-Phase Chemical Approach to a New Crystal Structure of Cobalt. Angew. Chem., Int. Ed. 1999, 38, 1788-1791.

(18) Puntes, V. F.; Krishnan, K. M.; Alivisatos, A. P. Colloidal Nanocrystal Shape and Size Control: The Case of Cobalt. Science 2001, 291, 2115-2117.

(19) van Schooneveld, M. M.; Campos-Cuerva, C.; Pet, J.; Meeldijk, J. D.; van Rijssel, J.; Meijerink, A.; Erné, B. H.; de Groot, F. M. F. Composition Tunable Cobalt-Nickel and Cobalt-Iron Alloy Nanoparticles Below $10 \mathrm{~nm}$ Synthesized Using Acetonated Cobalt Carbonyl. J. Nanopart. Res. 2012, 14, 991.

(20) Lagunas, A.; Jimeno, C.; Font, D.; Solà, L.; Pericàs, M. A. Mechanistic Studies on the Conversion of Dicobalt Octacarbonyl into Colloidal Cobalt Nanoparticles. Langmuir 2006, 22, 3823-3829.

(21) Wu, N.; Fu, L.; Su, M.; Aslam, M.; Wong, K. C.; Dravid, V. P. Interaction of Fatty Acid Monolayers with Cobalt Nanoparticles. Nano Lett. 2004, 4, 383-386.

(22) van Schooneveld, M. M.; Kurian, R.; Juhin, A.; Zhou, K.; Schlappa, J.; Strocov, V. N.; Schmitt, T.; de Groot, F. M. F. Electronic Structure of $\mathrm{CoO}$ Nanocrystals and a Single Crystal Probed by 
Resonant X-ray Emission Spectroscopy. J. Phys. Chem. C 2012, 116, $15218-15230$.

(23) Ghiringhelli, G.; Piazzalunga, A.; Dallera, C.; Trezzi, G.; Braicovich, L.; Schmitt, T.; Strocov, V. N.; Betemps, R; Patthey, L.; Wang, X.; et al. SAXES, a High Resolution Spectrometer for Resonant $\mathrm{X}$-ray Emission in the 400-1600 eV Energy Range. Rev. Sci. Instrum. 2006, 77, 113108.

(24) Strocov, V. N.; Schmitt, T.; Flechsig, U.; Schmidt, T.; Imhof, A.; Chen, Q.; Raabe, J.; Betemps, R.; Zimoch, D.; Krempasky, J.; et al. High-Resolution Soft X-ray Beamline ADRESS at the Swiss Light Source for Resonant Inelastic X-ray Scattering and Angle-Resolved Photoelectron Spectroscopies. J. Synchrotron Radiat. 2010, 17, 631643.

(25) Chen, C. T.; Idzerda, Y. U.; Lin, H. J.; Smith, N. V.; Meigs, G.; Chaban, E.; Ho, G. H.; Pellegrin, E.; Sette, F. Experimental Confirmation of the X-Ray Magnetic Circular Dichroism Sum Rules for Iron and Cobalt. Phys. Rev. Lett. 1995, 75, 152-155.

(26) Thornton, J. M. C.; Williams, R. H. An S/XPS Study of Hydrogen Terminated, Ordered Silicon (100) and (111) Surfaces Prepared by Chemical Etching. Phys. Scr. 1990, 41, 1047-1052.

(27) Dumas, P.; Chabal, Y. J.; Jakob, P. Morphology of HydrogenTerminated $\mathrm{Si}(111)$ and $\mathrm{Si}(100)$ Surfaces Upon Etching in $\mathrm{HF}$ and Buffered-HF Solutions. Surf. Sci. 1992, 269, 867-878.

(28) Ghiringhelli, G.; Brookes, N. B.; Annese, E.; Berger, H.; Dallera, C.; Grioni, M.; Perfetti, L.; Tagliaferri, A.; Braicovich, L. Low Energy Electronic Excitations in the Layered Cuprates Studied by Copper $\mathrm{L}_{3}$ Resonant Inelastic X-Ray Scattering. Phys. Rev. Lett. 2004, 92, 117406.

(29) Kotani, A.; Shin, S. Resonant Inelastic X-ray Scattering Spectra for Electrons in Solids. Rev. Mod. Phys. 2001, 73, 203-246.

(30) Ament, L. J. P.; van Veenendaal, M.; Devereaux, T. P.; Hill, J. P.; van den Brink, J. Resonant Inelastic X-ray Scattering Studies of Elementary Excitations. Rev. Mod. Phys. 2011, 83, 705-767.

(31) Hennies, F.; Pietzsch, A.; Berglund, M.; Föhlisch, A.; Schmitt, T.; Strocov, V.; Karlsson, H. O.; Andersson, J.; Rubensson, J.-E. Resonant Inelastic Scattering Spectra of Free Molecules with Vibrational Resolution. Phys. Rev. Lett. 2010, 104, 193002.

(32) Pietzsch, A.; Sun, Y. P.; Hennies, F.; Rinkevicius, Z.; Karlsson, H. O.; Schmitt, T.; Strocov, V. N.; Andersson, J.; Kennedy, B.; Schlappa, J.; et al. Spatial Quantum Beats in Vibrational Resonant Inelastic Soft X-Ray Scattering at Dissociating States in Oxygen. Phys. Rev. Lett. 2011, 106, 153004.

(33) van Schooneveld, M. M.; Juhin, A.; Campos-Cuerva, C.; Schmitt, T.; de Groot, F. M. F. Wet-Chemically Prepared Cobalt Bearing Nanoparticles Studied by 2p3d Resonant X-ray Emission Spectroscopy: a Probe of Surface-Adsorbate Bonds or Leached Metal Ions? Submitted.

(34) The absence of resonant Raman features has been observed by Prof. Dr. L. Braicovich (Dipartimento di Fisica, Politecnico di Milano, piazza Leonardo da Vinci 32, I-20133 Milano, Italy) on metal cobalt in early, unpublished 2p3d RXES experiments. Personal communication, 2012.

(35) Seah, M. P.; Dench, W. A. Quantitative Electron Spectroscopy of Surfaces: A Standard Data Base for Electron Inelastic Mean Free Paths in Solids. Surf. Interface Anal. 1979, 1, 2-11. 